\title{
Supported lipid bilayers as templates to design manganese oxide nanoparticles
}

\author{
J MAHESHKUMAR $^{\mathrm{a}}$, B SREEDHAR ${ }^{\mathrm{b}}$, B U NAIR $^{\mathrm{a}}$ and A DHATHATHREYAN ${ }^{\mathrm{a}, *}$ \\ ${ }^{a}$ Chemical Laboratory, CSIR-Central Leather Research Institute, Adyar, Chennai 600 020, India \\ bInorganic and Physical Chemistry Division, CSIR-Indian Institute of Chemical Technology, \\ Hyderabad 500 607, India \\ e-mail: aruna@clri.res.in
}

MS received 17 April 2012; revised 26 June 2012; accepted 30 July 2012

\begin{abstract}
This work reports on the preparation of nanoclusters of manganese oxide using biotemplating techniques. Supported lipid bilayers (SLBs) on quartz using cationic lipid [Dioctadecyldimethylammonium bromide (DOMA)] and mixed systems with neutral phospholipids dipalmitoyl phosphatidylcholine (DPPC) and dioleoyl phosphatidylcholine (DOPC) have been used as templates to synthesize these nanoparticles in a waterbased medium at room temperature. The Transmission electron microscopy (TEM) and Scanning electron microscopy (SEM) show manganese oxide nanostructures that are composed of crystals or small clusters in the size range of $20-50 \mathrm{~nm}$ in diameter. Small angle XRD showed that template removal through calcining process results in nanostructures of the manganese oxide in sizes from 30 to $50 \mathrm{~nm}$. Using these organized assemblies it is possible to control the nano and mesoscopic morphologies of particles and both rod-like and spherical particles can be synthesized.
\end{abstract}

Keywords. Manganese oxide; supported lipid bilayers; nanoparticles; organized assemblies.

\section{Introduction}

A variety of morphologies of nano-structured materials from many different facile synthetic methods have been reported in recent times. Among the different techniques, the templating method offers an original technique for designing advanced materials which allows control of nano or microstructures with specific functions. ${ }^{1}$ Template directed assemblies of organicinorganic structures include model bilayer membranes, vesicles and liposomes that have been used extensively to direct organization of the 2D structures. ${ }^{2-12}$ Among these soft templates, supported lipid bilayers (SLBs) have been gaining importance in recent times.

SLBs are of importance as a method of biofunctionalizing solid surfaces and can provide a model to investigate membrane proteins. They have been formed on micron sized spherical and nanoparticle surfaces, and have been investigated by molecular dynamics (MD) simulations. ${ }^{13-16}$ SLBs can be formed on planar and

*For correspondence curved surfaces by the fusion of small unilamellar vesicles (SUVs) on a variety of inorganic and organic supports. Fusion can occur by a process of adsorption and rupture, or by a single rupture step, and can depend on factors such as the ionic strength of the medium, type of buffer and solid support. ${ }^{17}$

Dynamics of SLB deposition from vesicle suspensions have been studied by Bucak et al. and the use of these suspensions to cover the surface of solid substrates have been evaluated. ${ }^{18}$ Dimova et al. have proposed a novel method of synthesizing nanoparticles in giant vesicles. ${ }^{19}$ Synthesis of nanoparticles in such bio-mimetic compartment allows for direct observation of induction, control and formation of the particles. ${ }^{20}$ Nanoparticles prepared using GUVs are isolated from the outside environment and thus can be prepared in a pure form. Further, the metal oxide nanoparticles synthesized inside vesicle is preferred mainly because of its morphology and large surface area. ${ }^{21}$ Such nanoparticles are highly stable against aggregation. Metal and metal oxide nanoparticles prepared using micro-reactors find application in catalysis, separation media and biopolymer tagging. ${ }^{22}$ Binding of charged nanoparticles to lipid bilayers, both as liposomes and substrate supported assemblies have 
been used to prepare nanocomposites by Goertz et al. ${ }^{23}$

Nanostructured metal oxides with different morphologies find application in a number of fields with their improved material characteristics like magnetic/ electronic properties and their enhanced catalytic/ optical performances that originate from their small size and extremely high specific surface area.

Among the many metal oxides, manganese oxides have received very less attention for utilization as anode materials, because of their limited capacity as a result of their extremely low electrical conductivity. ${ }^{24-27}$ They are attractive anode materials for lithium ion batteries because of the high abundance of $\mathrm{Mn}$, low cost, and environmental compatibility. We report here the synthesis and characterization of manganese oxide nanoclusters prepared using SLBs of cationic double tailed lipid Dioctadecyldimethylammonium bromide (DOMA), and mixed lipid systems containing DOMA and 1,2-dipalmitoyl-sn-glycero-3phosphocholine (DPPC) or dioleoyl phosphocholine (DOPC) as templates at room temperature. The SLBs have been formed using small unilamellar vesicles (SUVs) of the lipids containing $\mathrm{Mn}\left(\mathrm{NO}_{3}\right)_{2}$ which were then exposed to $\mathrm{KMnO}_{4}$. Figure 1 shows a schematic representation of the templated fabrication processes for manganese oxide nanostructures. The morphology and sizes of the as-synthesized products have been investigated using TEM, SEM and small angle XRD.

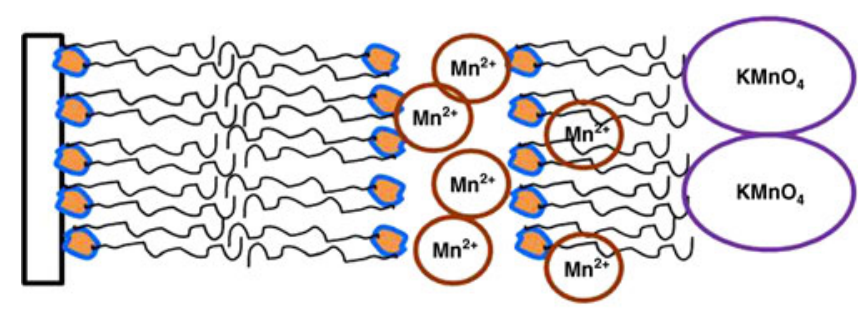

SLB of DOMA

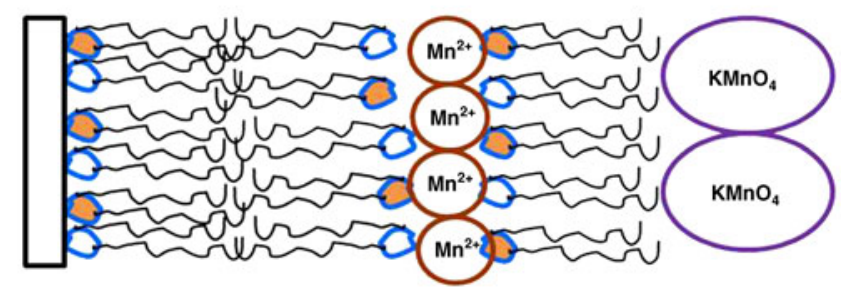

\section{SLB of DOMA+DPPC/DOPC}

Figure 1. Reaction of $\mathrm{Mn}\left(\mathrm{NO}_{3}\right)_{2}$ with $\mathrm{KMnO}_{4}$ in SLBs of DOMA and DOMA+DPPC.
Here, the manganese oxide was prepared using the reaction between $\mathrm{Mn}^{2+}$ and $\mathrm{MnO}_{4}^{-}$as follows

$$
2 \mathrm{MnO}_{4}^{-}+3 \mathrm{Mn}_{2}^{+}+2 \mathrm{H}_{2} \mathrm{O} \rightarrow 5 \mathrm{MnO}_{2}+4 \mathrm{H}^{+} .
$$

\section{Experimental}

DOMA, DPPC or DOPC obtained from Larodan Chemicals (Sweden) were $99.99 \%$ pure and used without further purification. All solutions/suspensions were prepared with Millipore-Milli-Q water. HPLC grade chloroform, Analar grade $\mathrm{KMnO}_{4}$ and $\mathrm{Mn}\left(\mathrm{NO}_{3}\right)_{2}$ were purchased from Merck India and used as such. An Avanti Mini-Extruder from Avanti Polar Lipids was used for extrusion of the lipids, using $50 \mathrm{~nm}$ polycarbonate filters.

\subsection{Supported lipid bilayers}

Defined amounts of lipids were dissolved in chloroform. Dry lipid films were formed after evaporation of the solutions under a stream of nitrogen and then in a vacuum oven overnight to remove any residual solvent. The lipid film was then redispersed in deionized water and incubated at a temperature above its transition temperature $T_{m}$ for a minimum of $2 \mathrm{~h}$ with periodic shaking to form hydrated multilamellar vesicles (MLVs). Small unilamellar vesicles (SUVs) were obtained from MLVs by subjecting them to 5 freeze/thaw cycles followed by extrusion using a polycarbonate filter with a pore size of $50 \mathrm{~nm}$. Approximately $1 \mathrm{ml}$ of a $5-10 \mathrm{mg} / \mathrm{ml}$ of the lipid solution was passed back and forth for up to 50 times. The vesicles became more monodisperse as the number of passes increased. Cleaned quartz slides have been used to form the SLBs. The slides have been washed in freshly prepared chromic acid, washed repeatedly with de-ionized distilled water and then sonicated in $n$-propylalcohol for $10 \mathrm{~min}$. For each measurement freshly cleaned slides have been used after storing them in desiccator for $3 \mathrm{~h}$. These slides have been cleaned in a Plasma Cleaner (Harrick Plasma, USA) before coating with two layers of the lipid DOMA, DOMA+DPPC or DOMA+DOPC to ensure the surface hydrophilicity.

\subsection{Preparation of SLBS}

The 2 layer LB film-coated slide was placed in a custom-built flow-through chamber, and vesicles containing $\mathrm{Mn}\left(\mathrm{NO}_{3}\right)_{2}$ were injected and incubated with the surface for 30-60 min (pure lipid vesicles) and gently shaken in a water bath shaker (REMI, India). Excess 
vesicles were washed out by extensive rinsing with buffer. $\mathrm{KMnO}_{4}$ was introduced to these slides in an airtight compartment after which they were subjected to calcinations at $\mathrm{T}=200^{\circ} \mathrm{C}$.

\subsection{Transmission electron microscopy and Scanning electron microscopy (TEM and SEM)}

For the TEM studies similar procedures were repeated with Formvar/carbon coated copper grids (Mesh size 200) using TECNAI FE12 TEM instrument operating at $120 \mathrm{kV}$ using SIS imaging software. For the SEM analysis, samples were prepared on glass substrates by the above procedure and a thin-layer of gold $(200 \AA)$ was sputtered on these samples and scanning electron microscopic studies were undertaken using a Hitachi SU1510 model.

\subsection{XRD study}

Small angle XRD studies on the calcined sample to identify the different phases and crystal packing of the $\mathrm{MnO}_{2}$ were carried out using SEIFERT -JSO DEBYEFLEX 2002, FRG. The formation of SLBs and their thickness were evaluated using SLB coated $2.2 \times 2.2 \mathrm{~cm}^{2}$ thin-glass cover slips (ERMA, FRG) cleaned with isopropyl alcohol and chloroform using XRD measurements. These samples are ideal for Xraying through the back of the glass at a $45^{\circ}$ angle of incidence; the scattering from the sample is then totally unobstructed by the substrate. These coated cover slips were attached to the cap of a $20 \mathrm{~mL}$ disposable glass scintillation vial using either sticky tack or clay. For all the X-ray data each scan covered $\theta$ from $0.5^{\circ}$ to $5^{\circ}$ with step size $0.005^{\circ}$, one second per step. The humidity/temperature chamber enclosed the goniometer head insulated from room temperature. The temperature of the sample was monitored by a Pt-100 thermo resistor and controlled to $\pm 0.025^{\circ} \mathrm{C}$ via a computer based feedback system.

\section{Results and discussion}

The thickness of SLBs using small angle XRD shows an average thickness of about $64 \AA$ for the pure DOMA films and 58-60 $\AA$ for the mixed films of DOMA with DOPC or DPPC which is in agreement with the lamellar spacing for the bilayers obtained from molecular models.

Figures 2 (a), (b) and (c) show the SEM micrographs of the oxide obtained from SLBs of DOMA,
DOMA+DPPC and DOMA+DOPC. In SLBs formed using DOMA, close examination reveals that the nanoparticles formed seem to be just clusters of beads of average size $\sim 10-15 \mathrm{~nm}$. Since DOMA is cationic, normally due to electrostatic repulsion between the neighbouring positively charged headgroups in the polar plane, the sizes of manganese oxide formed should be small and have a narrow size distribution. However, due to the large amount of water that is entrained in the head group, on drying the spherical clusters seem to aggregate. Interfacial water structure

(a)

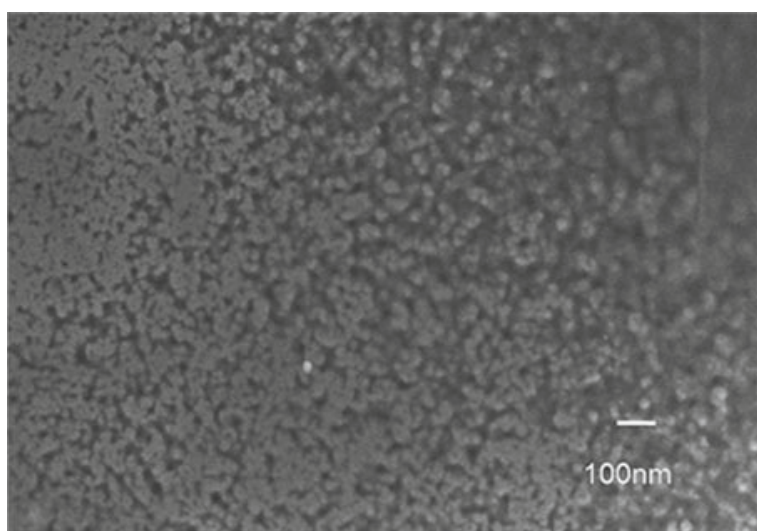

(b)

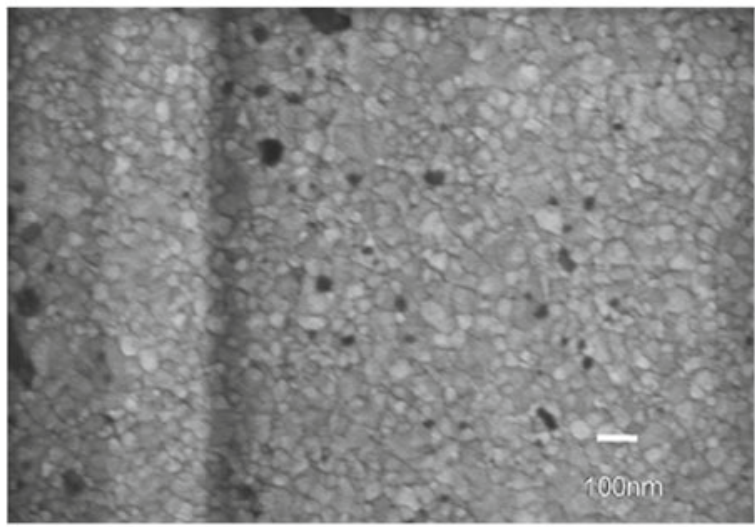

(c)

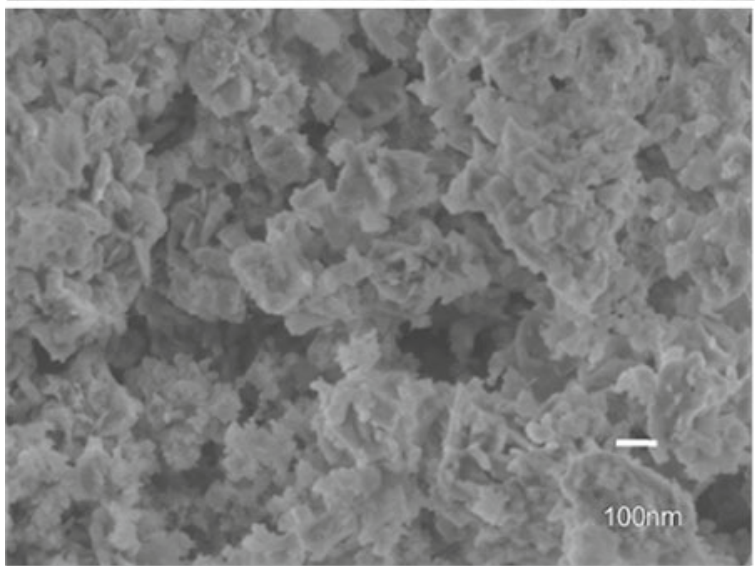

Figure 2. SEM micrographs of manganese oxide formed from SLBs of (a) DOMA, (b) DOMA+DPPC and (c) DOMA+DOPC. 
associated with different head groups in Langmuir monolayers containing pure DOMA and DOMA with octadecylamine was reported by Nikolov et al. ${ }^{28}$ In this work, it was demonstrated that 'electric field induced' highly ordered water structure and some fraction of weakly $\mathrm{H}$-bonded loosely bound water molecules exist near the cationic head groups of DOMA. Thus, SLBs of DOMA have a tendency to abstract more water from the hydrated manganese ions which in turn lead to stronger clustering of the metal ions.

(a)

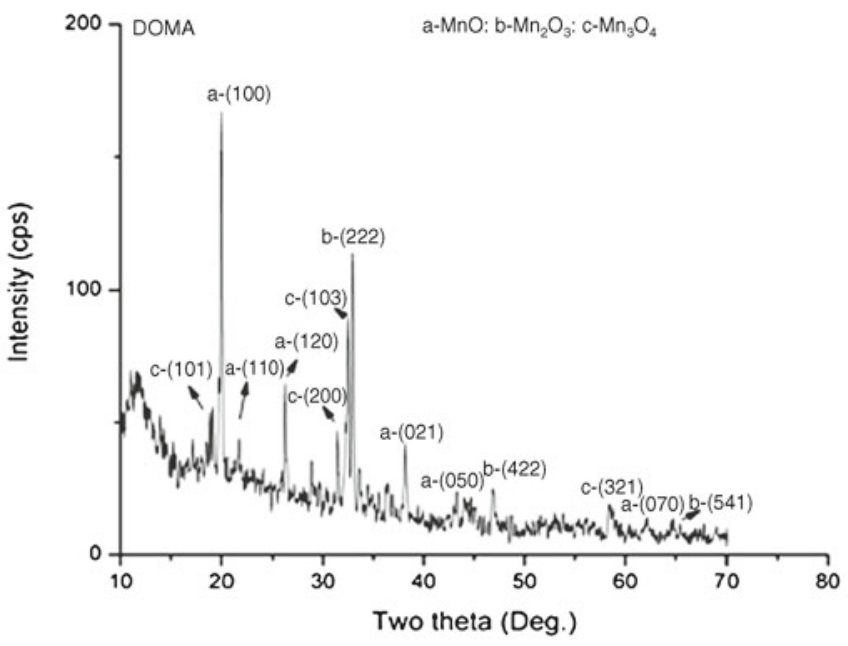

(b)

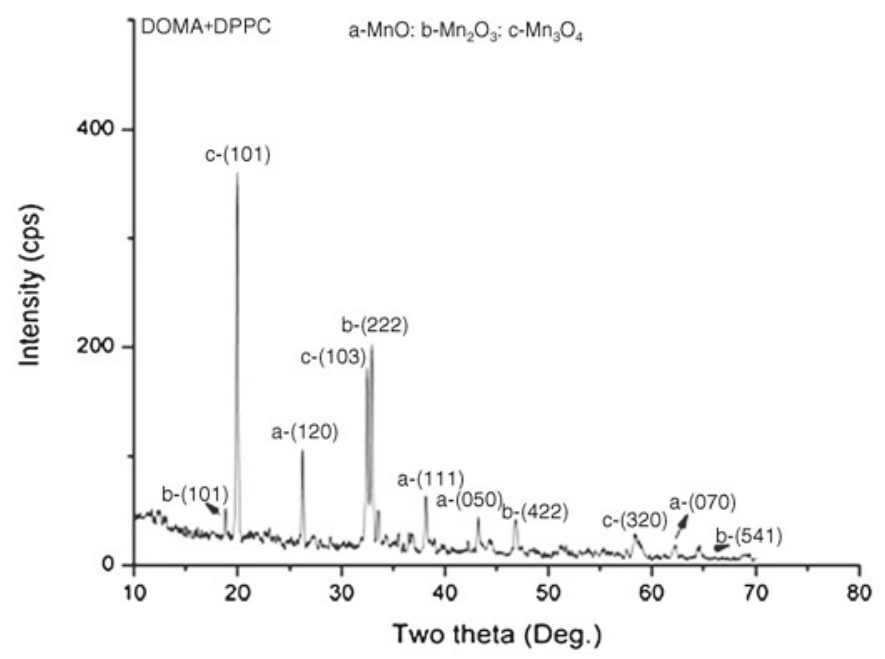

(c)

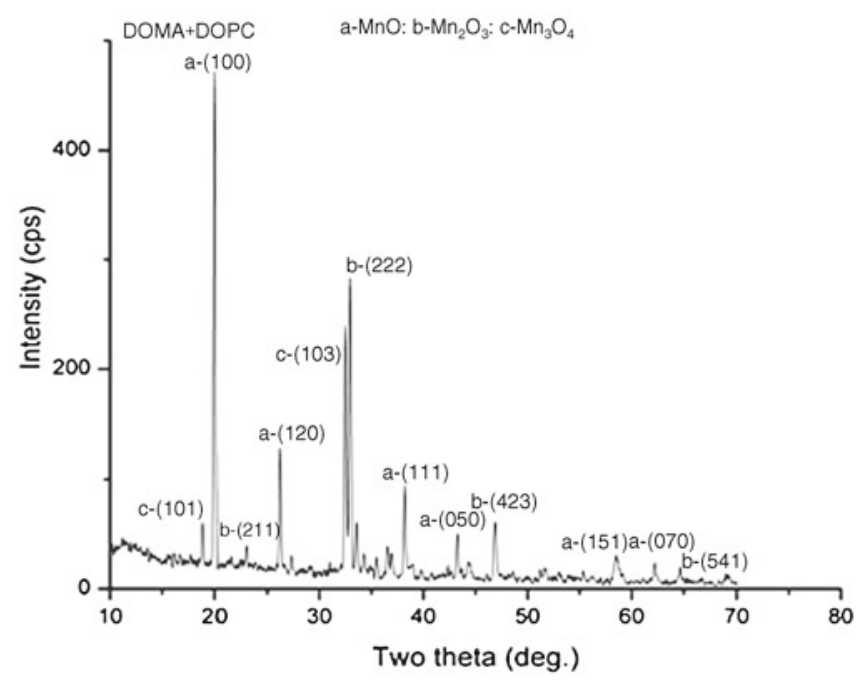

Figure 3. Powder XRD patterns of manganese oxide formed from SLBs of (a) DOMA, (b) DOMA+DPPC and (c) DOMA+DOPC. 
In SLBs of mixed systems of DOMA+DPPC or DOMA+DOPC it is observed that either platelets or nanostrips of average size $50 \mathrm{~nm}$ to $100 \mathrm{~nm}$ is seen, which are larger in size compared to samples obtained from pure DOMA template. Thus, dilution of cationic charges with the DOPC or DPPC prevents small clusters from forming and also results in larger aggregates. The crystallinity and phase composition of the final products investigated by X-ray powder diffraction are presented in figure 3 (a), (b) and (c) using DOMA, DOMA+DPPC and DOMA+DOPC as templates. A comparison with the JCPDS data suggests that a mixture $\mathrm{MnO}, \mathrm{Mn}_{2} \mathrm{O}_{3}$ and $\mathrm{Mn}_{3} \mathrm{O}_{4}$ is obtained. This is possibly due to the fact that on standing the SLBs can locally show small changes in interfacial $\mathrm{pH}$ which can lead to both reaction between soluble $\mathrm{MnO}_{4}^{-}$and $\mathrm{Mn}_{2}^{+}$and also reduction of $\mathrm{MnO}_{4}^{-}$by water. Thus, mixed oxidation states of $+3 /+4$ of manganese is possible. In addition, Calcination of the SLBs containing the alkyl chains leads to carbonaceous material which also reduces the manganese oxide to $\mathrm{MnO}$.

In figure 3 (a), all strong peaks are undoubtedly indexed to a face-centred cubic $\mathrm{MnO}$ phase, but a mixture of $\mathrm{MnO}$ as the dominant phase and $\mathrm{Mn}_{2} \mathrm{O}_{3}$ and $\mathrm{Mn}_{3} \mathrm{O}_{4}$ as the minor components. According to the XRD pattern, a simple comparison of the intensity of the strongest lines, the (100) reflection at $2 \theta=$ $20.01^{\circ}$ of $\mathrm{MnO}$ and the (222) reflection at $2 \theta=32.75^{\circ}$ of $\mathrm{Mn}_{2} \mathrm{O}_{3}$, clearly supports this finding. The intensity of the $\mathrm{MnO}$ peak is much higher than the one of $\mathrm{Mn}_{2} \mathrm{O}_{3}$ or $\mathrm{Mn}_{3} \mathrm{O}_{4}$ implying a considerably higher content of $\mathrm{MnO}$ in the product. Similarly, templates of DOMA+DPPC and DOMA+DOPC as SLBs (figure 3 $b, c)$ showed that the product formed was a mixture of all the oxides. In case of DOMA+DPPC the dominant fraction is $\mathrm{Mn}_{3} \mathrm{O}_{4}$ while with DOMA+DOPC it is $\mathrm{MnO}$. We observe that with DOMA SLB, there is more broadening of the peaks compared with the other two templates. This could arise from both instrumental broadening of the diffraction profiles as well as small size of the nano clusters.

Using the Debye Scherer formula

$$
\begin{aligned}
\text { Particle size }= & (0.9 * \lambda) /(\mathrm{FWHM} * \cos \theta) \\
& \text { can be estimated. }
\end{aligned}
$$

The average crystallite sizes calculated for the dominant species are $24.5 \mathrm{~nm}$ (DOMA), $35.9 \mathrm{~nm}$ (DOMA+DPPC) and $33.5 \mathrm{~nm}$ (DOMA+DOPC). Generally, the average crystallite sizes are expressed in terms of different directions in reciprocal space. However, in the present case the line broadening is hkl independent (isotropic), making the labelling of the average crystallite size with hkl superfluous. The average crystallite size of $\mathrm{MnO}$ equals $24.5 \mathrm{~nm}$, which is considerably smaller than that of the $\mathrm{MnO}$ sample, obtained using DOMA+DOPC (figure 3).

Transmission Electron Microscopy (TEM) was performed on the three samples by ultrasonically dispersing them and then transferring to the TEM grid. Representative TEM images of the manganese oxide nanoparticles are displayed in figure $4 \mathrm{a}$ and $\mathrm{c}$

(a)

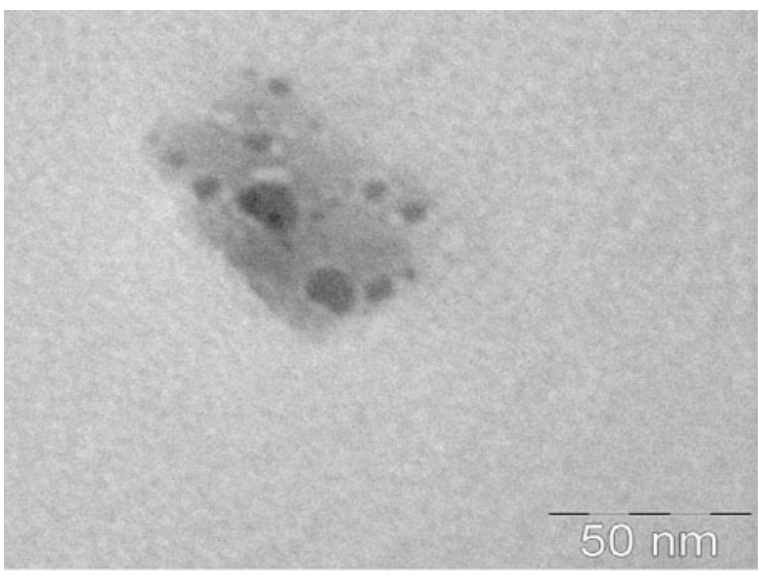

(b)

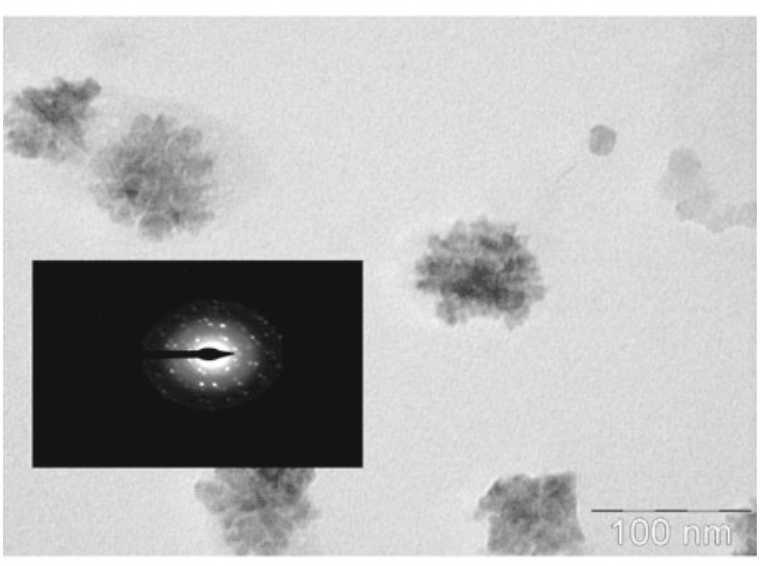

(c)

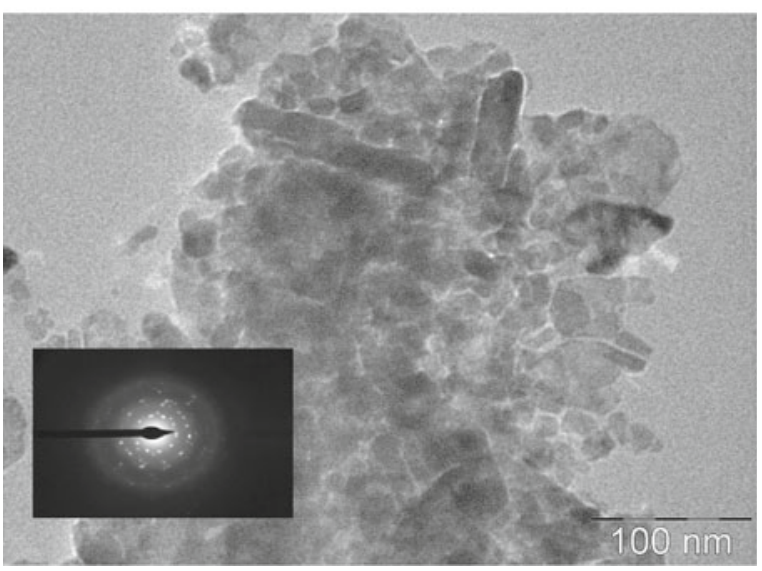

Figure 4. TEM micrographs of manganese oxide formed from SLBs of (a) DOMA, (b) DOMA+DPPC and (c) DOMA+DOPC. 
with (MnO as dominant phase in DOMA and DOMA+DOPC) and figure $4 \mathrm{~b}\left(\mathrm{Mn}_{3} \mathrm{O}_{4}\right.$ as dominant phase), clearly proving that the obtained samples are composed of well-defined nanoparticles with a lowdegree of agglomeration.

Comparison of the average crystallite size calculated from XRD with the average diameters extracted from TEM images reveals that the nanoparticles are as a matter of fact composed of single crystals in the nanometric size region. The small discrepancy between the average values from XRD and TEM can be attributed to the limited statistical assembly for TEM (100 particles) introducing higher uncertainties in the calculation of the average values. The phase composition deduced from XRD analysis is further confirmed by SAED. For the $\mathrm{Mn}_{3} \mathrm{O}_{4}$-dominated sample, the reflections of both compounds are well-resolved in the XRD pattern. However, in the MnO-dominated sample the 101 reflection of $\mathrm{Mn}_{3} \mathrm{O}_{4}$ is not clearly observed.

\section{Conclusion}

In conclusion, The SLBs with cationic lipid DOMA as template with $\mathrm{Mn}\left(\mathrm{NO}_{3}\right)_{2}$ reacting with $\mathrm{KMnO}_{4}$ yield a phase mixture of manganese (II) oxide and manganese (II, III) oxide as the minor component. The analogous reaction with mixed SLBs from DOMA+DPPC results in the inverse situation, where manganese (II, III) oxide represents the main and manganese (II) oxide the minor component. Among the different parameters, change in the $\mathrm{pH}$ near the head groups of the lipids in the SLBs during the aging process, counter-cation nature and concentration, seem to control the Mn oxidation state between various polymorphs. The proper choice of the above parameters in the SLB template should provide a handle to design manganese oxides as nanoparticles with various shapes, including nanoplatelets, spherules and ellipsoids.

\section{Acknowledgements}

The authors thank the Department of Science and Technology (DST) project of DST-nanomission for a grant under which part of the work was carried out. MJ thanks DST-Innovation in Science Pursuit for Inspired Research (INSPIRE) program, Government of India for a research fellowship.

\section{References}

1. Cheng F, Tao Z, Liang J and Chen J 2008 Chem. Mater. 20667

2. Zhou H, Fan T, Zhang D, Guo Q and Ogawa H 2007 Chem. Mater. 192144

3. Shim H W, Jin Y H, Seo S D, Lee S H and Kim D W 2011 ACS Nano 5443

4. Royston E, Ghosh A, Kofinas P, Harris M T and Culver J N 2008 Langmuir 24906

5. Bharde A A, Parikh R Y, Baidakova M, Jouen S, Hannoyer B, Enoki T, Prasad B L, Shouche Y S, Ogale S and Sastry M 2008 Langmuir 245787

6. Nam K T, Kim D W, Yoo P J, Chiang C Y, Meethong N, Hammond P T, Chiang Y M and Belcher A M 2006 Science 312885

7. Lee Y J, Yi H, Kim W J, Kang K, Yun D S, Strano M S, Ceder G and Belcher A M 2009 Science 3241051

8. Walde P 1996 Curr. Opin. Colloid Interface Sci. 1638

9. Hotani H, Nomura F and Suzuki Y 1999 Curr. Opin. Colloid Interface Sci. 4358

10. Wick R, Angelova M I, Walde P and Luisi P L 1996 Chem. Biol. 3105

11. Tsumoto K, Nomura S-I M, Nakatani Y and Yoshikawa K 2001 Langmuir 177225

12. Kyotani T, Tsai L and Tomita A 1996 Chem. Mater. 8 2109

13. Liang Q and Ma Y Q 2009 J. Phys. Chem. B 1138049

14. Fabre R M and Talham D R 2009 Langmuir 2512644

15. Xing C and Faller R 2008 J. Phys. Chem. B 1127086

16. Yeh I C and Wallqvist A 2011 Biophys. J. 100 150a

17. Johnson J M, Ha T, Chu S and Boxer S G 2002 Biophys. J. 833371

18. Bucak S, Wang C, Laibinis P E and Hatton T A $2010 \mathrm{~J}$. Colloid Interface Sci. 348608

19. Yang P, Dimova R, 2011 in: Biomimetic based applications, George A (ed.) (InTech Publisher) p. 523

20. Yang P, Lipowsky R and Dimova R 2009 Small 52033

21. Klasczyk B, Knecht V, Lipowsky R and Dimova R 2010 Langmuir 2618951

22. Dimova R, Aranda S, Bezlyepkina N, Nikolov V, Riske K A and Lipowsky R 2006 J. Phys.: Condens. Matter. $18 \mathrm{~S} 1151$

23. Goertz M P, Goyal N, Bunker B C and Montaño G A 2011 J. Coll. Interf. Sci. 358635

24. Fan Q and Whittingham M S 2007 Electrochem. SolidState Lett. 10 A48

25. Pasero D, Reeves N and West A R 2005 J. Power Sources 141156

26. Wang H, Chi L, Yang Y and Casalongue H S $2010 \mathrm{~J}$. Am. Chem. Soc. 13213978

27. Shim H W, Lim A H, Min K M and Kim D W 2011 Cryst. Eng. Commun. 136747

28. Nickolov Z S, Britt D W and Miller J D 2006 J. Phys. Chem. B 11015506 